\title{
Impact of The Number of Light Emitting Diode Towards The Accuracy in Indoor Positioning System Based on Visible Light Communication
}

\author{
Brian Pamukti*, Miftakhul Meiliana Rahmawati, Nachwan Mufti Adriansyah \\ Department of Electrical Engineering \\ Telkom University \\ Jl. Telekomunikasi No. 1, Terusan Buah Batu, Sukapura, Dayeuh Kolot \\ Bandung 40257, Indonesia
}

\begin{abstract}
This research detects the random position with received signal strength (RSS) method based on the received power in the room with a size of $5 \times 5 \times 3$ cubic meter and several numbers of light emitting diode (LEDs). The first scenario uses 4 LEDs, the second scenario uses 6 LEDs and the third scenario uses 8 LEDs. Random points as the detector are placed spread at the room as many as 25 points. The simulation result shows that the use of more LEDs reduces the positioning error and increases the accuracy. If the accuracy reduces, the positioning error increase and affect the detection results. Using 8 LEDs, the number of the detected random point increases $30 \%$, the positioning error is $47 \%$, and the accuracy increase from $33 \%$. In addition, our research is useful for finding the locations of small items such as sensors that are scattered in a closed room.
\end{abstract}

Keywords: visible light communication, light emitting diode, received signal strength, accuracy, positioning error.

\section{INTRODUCTION}

Visible light communication (VLC) is a media communication that uses visible light as the light source to transmit information. VLC is often focused on indoor applications. The indoor positioning system based on VLC is more accurate than the global positioning system (GPS) and useful to obtain an object position with small size [1]. The differences in the number of LED used in the system can affect the detection results.

The research in [2] explains the indoor positioning system using differential detection. The result of the positioning system with differential detection has been compared with the positioning system with the Received Signal Strength (RSS) algorithm. The RSS algorithm obtained the detector's position based on the received power. The research used 3 LEDs and 2 detectors on the system. When one detector's position is known, the other detector's position can be obtained. With differential detection, the deviation of the measured results for each point is less than with the RSS algorithm and the average error is significantly reduced. The proposed positioning method can be used as high precision, high stability, and large area positioning in an actual LED lighting environment.

Researchers in [3] explain indoor location estimation based on the RSS algorithm using radial log-normal distribution. The researchers estimate the location of the object using marginal likelihoods of the radial lognormal distribution.

\footnotetext{
* Corresponding Author

Email: brianp@telkomuniversity.ac.id

Received: October 02, 2020 ; Revised: November 25, 2020

Accepted: December 10, 2020 ; Published: December 31, 2020

(C) 2020 PPET - LIPI
}

They compare the estimation accuracy of the static case and dynamic case. With a static case experiment, the data set when the object with the receiver keeps stopping within a minute on the fixed point. With a dynamic case experiment, the data set when the object with the receiver moves on the prescribed trajectory. Based on the experiment results, the static case is more be able to estimate location precisely. In the next phase, they need to improve the proposed method for the object movement case.

In another study [4],the combination of a simple regression-based approach with linear least square (LLS) and non-linear least square (NLS) estimation is explained. The researchers also explain the use of regression technique in order to get an estimated distance closer to the real distance. The regression is applied to all the rooms with 676 points and on the outer region with 352 points. They compared the localization error distribution using both LLS and NLS with and without regression. From the results, the regression-based approach significantly improves the localization accuracy. This approach achieves an average error of only $22 \mathrm{~cm}$ with $99.4 \%$ of the room having localization errors less than $0.6 \mathrm{~m}$.

The method to predict the location of sensors or robots has been carried out in [5]. The study conducted experiments with methods similar to algorithms on fingerprints. In addition, based on the Bayesian method the accuracy is increased. This study has a deficiency in the relatively small accuracy value so that the positioning error obtained is still large.

Multiplexing technology has been widely used for wireless communication media. With multiplexing, bandwidth is more efficient in the data transmission 
process. Researcher in [6] has published an indoor positioning system combined with multiplexing. The multiplexing pattern used is ACO-OFDM. In the application of ACO-OFDM, it has the disadvantage of cutting the symbol due to the unipolar type of optical transmission.

Research on visible light positioning (VLP) has also been carried out in [7]. With VLP research, it is hoped that it can be more accurate and cheaper when compared to adding an access point to wireless fidelity (WiFi) or satellite. This study uses a three-dimensional (3D) approach to obtain better accuracy. This study resulted in a low positioning error of up to $7.95 \mathrm{~cm}$. However, the space used is too small because it uses $2.5 \mathrm{~m} \mathrm{x} 2.5 \mathrm{~m} \mathrm{x} 3$ $\mathrm{m}$.

A few studies focused on increases the accuracy with a different method. There is no researcher concerned about the impact of the LED number uses in the system. Therefore, this research focused on the number of the LED. In this paper, we analyze the indoor positioning system using the RSS algorithm. We use a room with a size of $5 \times 5 \times 3 \mathrm{~m}$ and several numbers of LEDs $(4,6$ and 8 ) with a power of 1 watt. Also, we use 25 random points as the detector are placed spread at the room. To prove our research, we compared the estimated location with the real location. Based on the estimation, we show the accuracy and positioning errors.

The rest of this paper are organized as follows, in section II we describe the theory of the research followed by the description of the system model of the positioning system in in section III. In section IV we calculate all the formula using computer simulation for each signal format and compare its performance against the parameters system, such as random point detected positioning error and accuracy. Finally, we show the conclusion of this paper in section $\mathrm{V}$.

\section{METHOD}

\section{A. Line of Sight Channel}

In a Line of sight (LOS) channel the communication path between the transmitter to the receiver does not have an obstacle, so it can be said to be direct communication. The LOS condition allows the communication system to have no interference, so the quality of communication is relatively better. The LOS equation is shown below: [8]

$$
H(0)=\left\{\begin{array}{cc}
\frac{m+1}{2 \pi d^{2}} \operatorname{Acos}^{m}(\phi) T_{s}(\varphi) g(\varphi) \cos (\varphi), & 0 \leq \varphi \leq \varphi_{c} \\
0 & \varphi>\varphi_{c}
\end{array}\right.
$$

with $\Phi$ is the irradiance angle, $\varphi$ is the incidence angle, $T_{S}(\varphi)$ is the optical filter gain, $g(\varphi)$ is the optical concentrator gain, $\varphi_{c}$ is the field of vision at a receiver, and $m$ is the order of Lambertian emission. The difference in power between 0 and 1 is $P_{\text {diff, while the }}$ difference in received power is defined as

$$
P_{\text {diff }}=H(0) P_{\text {diff }}
$$

and the distance $d$ is defined in equation (3).

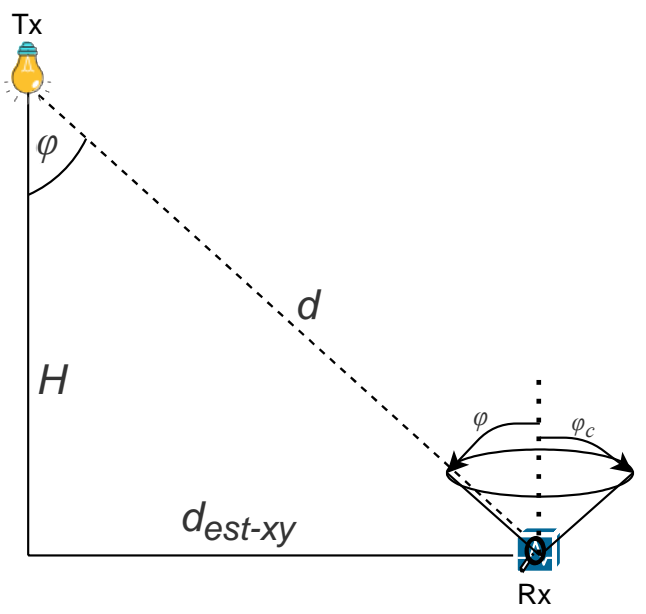

Figure 1. Line of sight channel model for visible light communication.

$$
\begin{gathered}
d^{2}=\frac{(m+1) A \cos ^{m}(\phi) T(\varphi) g(\varphi) \cos (\varphi) P \text { diff }}{2 \pi P_{\text {diff }}} \\
d_{\text {est }-x y}^{2}=d^{2}-H^{2}
\end{gathered}
$$

LOS conditions can be described as shown in Figure 1.

\section{B. Distance of Transmitter and Receiver}

In electromagnetics, the relationship between $d$ and the receiving power is defined as

$$
d=10^{\frac{P_{r}-P_{1 m}}{-10 . n}}
$$

where $P_{r}$ is the received power, $P_{I m}$ is the received power at 1 meter and $n$ is the exponent path loss. In the VLCbased positioning system, based on the received power at the detector, the value of $d$ can be defined as

$$
d=\sqrt[m+4]{\frac{(m+1) \cdot A_{d e t} \cdot h^{m+1}}{H \cdot 2 \pi}}
$$

\section{RSS Algorithm}

The RSS method is used more frequently in indoor positioning systems because of its low difficulty and high accuracy. In the principle of detection using RSS, the system will calculate the distance between the LED and the detector based on the value of the received power, then the position coordinates of the detection target can be formulated with at least 2 equations as [8]

$$
\begin{gathered}
{\left[\begin{array}{l}
x \\
y
\end{array}\right]=A^{-1} B} \\
A=\left[\begin{array}{ll}
2\left(x_{2}-x_{1}\right) & 2\left(y_{2}-y_{1}\right) \\
2\left(x_{3}-x_{1}\right) & 2\left(y_{3}-y_{1}\right)
\end{array}\right] \\
B=\left[\begin{array}{l}
d_{1}^{2}-d_{2}^{2}-\left(x_{1}^{2}+y_{1}^{2}\right)-\left(x_{2}^{2}+y_{2}^{2}\right) \\
d_{1}^{2}-d_{3}^{2}-\left(x_{1}^{2}+y_{1}^{2}\right)-\left(x_{3}^{2}+y_{3}^{2}\right.
\end{array}\right]
\end{gathered}
$$


where $d$ is the distance of LED and detector, $x$ and $y$ are the coordinates of LED. The RSS algorithm requires at least three distances from an access point. However, using three lights still has a very high probability of error. Therefore, we experimented with the RSS algorithm for several lamps.

\section{Positioning Error}

Positioning error is one of the important parameters from the detection results of VLC positioning. When the number of LEDs used in the system increases, the number of positioning errors is also increased. Positioning error can be formulated as [2]

$$
D=\sqrt{\left(x_{r}-x_{m}\right)^{2}+\left(y_{r}-y_{m}\right)^{2}}
$$

where $\left(x_{r}, y_{r}\right)$ is the real coordinates of the random points and $\left(x_{m}, y_{m}\right)$ is the detected coordinates of the random points.

\section{E. Accuracy}

Accuracy is important for the validation of the simulations being carried out. We obtain the accuracy from the difference between the original coordinates and the detection results. We use the computation of the Rscore which makes the difference of squares of the differences in the comparable data. Accuracy is the main parameter of the detection results of VLC positioning. The higher the accuracy, the better the system. Accuracy can be formulated as

$$
R^{2}=1-\frac{\sum_{i}\left(y_{i}-f_{i}\right)^{2}}{\sum_{i}\left(f_{i}-\bar{y}\right)^{2}}
$$

where $y_{i}$ is the real position, $f_{i}$ is the detected position and $y$ is the average of the real position.

\section{F. Research Design}

The detector receives the power sent by the LED, then the distance $(d)$ between the transmitter and receiver can be determined, which is not less than the $h$. Moreover, the $(x, y)$ coordinates of the random point can be determined based on the calculated $d$. After the coordinates $(x, y)$ are determined, the positioning error value is calculated.

\section{1) Room Design with 4 LEDs}

The first scenario uses 4 LEDs in a room with a size of $5 \mathrm{~m} \times 5 \mathrm{~m} \times 3 \mathrm{~m}$. Figure 2 shows the location of the LED at coordinates of $(2.5,2.5,3),(2.5,-2.5,3),(-2.5,-2.5,3)$ and $(-2.5,2.5,3)$, with the random point receiver locations are spread over room. The detection method used is the RSS algorithm. If the simulation results achieve a coordinate accuracy of more than $70 \%$, the simulation is complete.

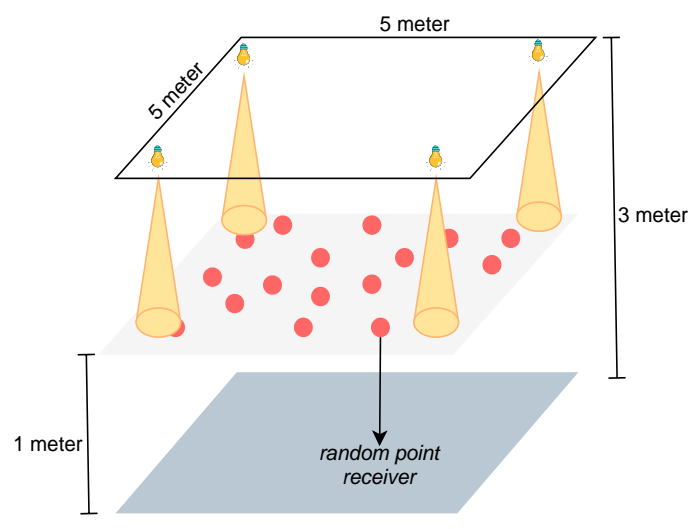

Figure 2. System model using 4 LEDs.

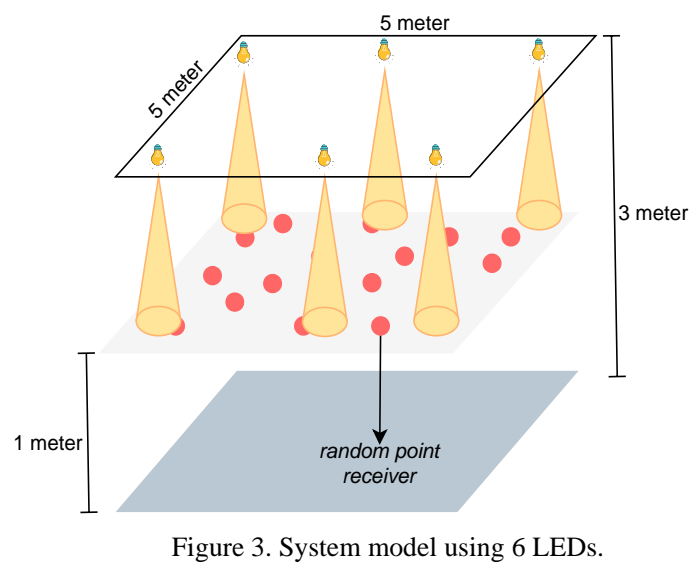

2) Room Design with 6 LEDs

The second scenario uses 6 LEDs in a room with a size of $5 \mathrm{~m} \times 5 \mathrm{~m} \times 3 \mathrm{~m}$. Figure 3 shows the location of the LED at coordinates of $(2.5,2.5,3),(2.5,0,3),(2.5,-2.5$, $3),(-2.5,-2.5,3),(-2.5,0,3)$ and $(-2.5,2.5,3)$, with the random point receiver locations are spread over room. The detection method used is the RSS algorithm. If the simulation results achieve a coordinate accuracy of more than $70 \%$, the simulation is complete.

\section{3) Room Design with 8 LEDs}

The last scenario uses 8 LEDs in a room with a size of $5 \mathrm{~m} \times 5 \mathrm{~m} \times 3 \mathrm{~m}$. Figure 4 shows the location of the LED at coordinates of $(2.5,2.5,3),(2.5,0,3),(2.5,-2.5,3),(0$, $-2.5,3),(-2.5,-2.5,3),(-2.5,0,3),(-2.5,2.5,3)$ and $(0$, $2.5,3)$, with the random point receiver locations are spread over room. The detection method used is the RSS algorithm. If the simulation results achieve a coordinate accuracy of more than $70 \%$, the simulation is complete.

TABLE 1: SIMULATION PARAMETERS.

\begin{tabular}{|l|l|}
\hline \multicolumn{1}{|c|}{ Parameter } & \multicolumn{1}{c|}{ Value } \\
\hline Size of room & $5 \mathrm{~m} \times 5 \mathrm{~m} \times 3 \mathrm{~m}$ \\
\hline Type of source & LED \\
\hline Optical power of LED & $1 \mathrm{Watt}$ \\
\hline Amount of LED & $4,6,8$ \\
\hline Type of photodetector & P-I-N \\
\hline Amount of photodetector & 25 \\
\hline The physical area of detector & $1 \mathrm{~cm}^{2}$ \\
\hline Height between the LED and detector & $2 \mathrm{~m}$ \\
\hline
\end{tabular}




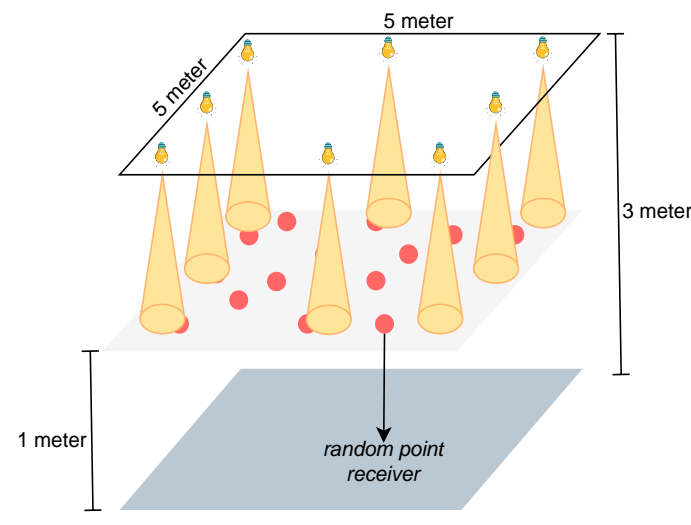

Figure 4. System model using 8 LEDs.

\section{G. Simulation Parameters}

Table 1 describes the parameters of the simulation. In this research, we use LED as the light source to transmit data with the number of LEDs of 4, 6 and 8, and with $1 \mathrm{~W}$ transmission power. LOS propagation model of the channel is used in this research. We also use a PIN photodetector as a receiver. PIN selection is used in this simulation because of its relatively inexpensive installation on small devices such as sensors. In addition, we placed the receiver 1 meter above the floor so that the height from the LED to the receiver is 2 meters.

\section{RESUlt AND Discussion}

\section{A. Performance of Random Point Detection}

We simulate the indoor positioning system with the number of LEDs of 4, 6 and 8. The simulation uses 25 random points for the detection targets and receivers. Based on the results, the system with 8 LEDs has a better amount of random point detection than that of 4 and 6 LEDs. This is because the light coverage produced by 8 LEDs is the widest than the 4 and 6 LEDs.

Figure 5 shows the comparison of the detection results of the receiver position and the original receiver position using 4 LEDs. With 4 LEDs, the random points detected are 17 out of 25 . From the results of extensive simulations, we found that with 4 LEDs configuration the detection value was more sufficient by the triangulation method. However, the value of the detection success score is around $67 \%$. This result is not enough, because there are many coordinates that are similar even though they are in different places. 4 LEDs scenario is actually sufficient for the RSS algorithm, because it only takes at least 3 LEDs to triangulate. However, the result shows that the experiment of 4 LEDs cannot detect all 25 users in various locations accurately $100 \%$. We obtain a small positional shift from the actual location.

Figure 6 shows the comparison of the detection results of the receiver position and the original receiver position using 6 LEDs. With 6 LEDs, the random points detected are 22 out of 25 . From the results of extensive simulations, we found that with 6 LEDs configuration, the detection value was sufficient by the triangulation method. However, the value of the detection success score is around $88 \%$. This result is insufficient, because there are many coordinates that are similar even though they are in different places.

Figure 7 shows the comparison of the detection results of the receiver position and the original receiver position using 8 LEDs. With 8 LEDs configuration, the random points detected are 24 out of 25 . From the results of extensive simulations, we found that with 8 LEDs the detection value was sufficient by the triangulation method. However, the value of the detection success score is around $96 \%$. This result is satisfactory, because there are many coordinates that are similar with the real coordinate of receivers.

\section{B. Performance of Positioning Error}

We evaluate the results of the positioning error simulation for scenarios 4, 6 and 8 LEDs. Positioning error affects the accuracy of the simulation. The higher number of LEDs are used, the smaller the number of positioning errors, which means the system is getting better. This can be seen in Figure 5 which shows the comparison of the positioning error on the 4,6 and 8 LEDs scenarios.

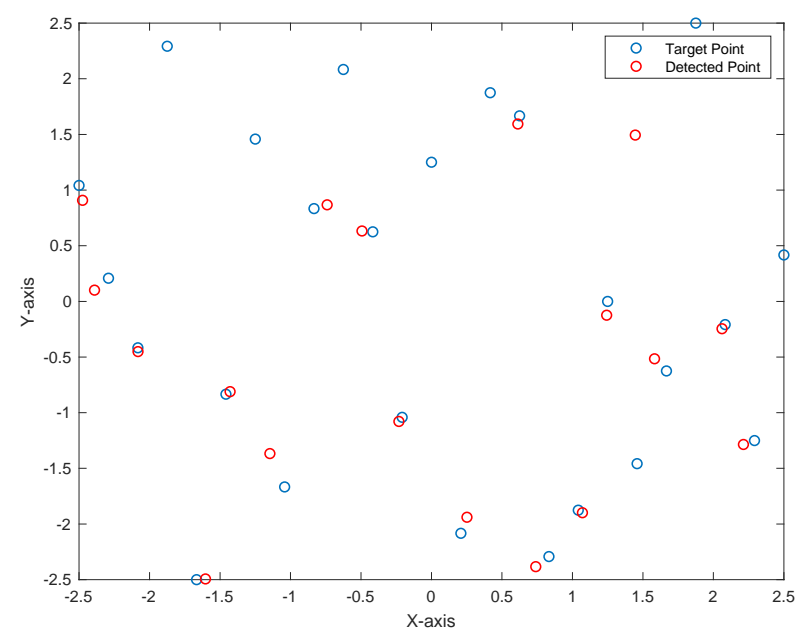

Figure 5. Result of random point detection using 4 LEDs.

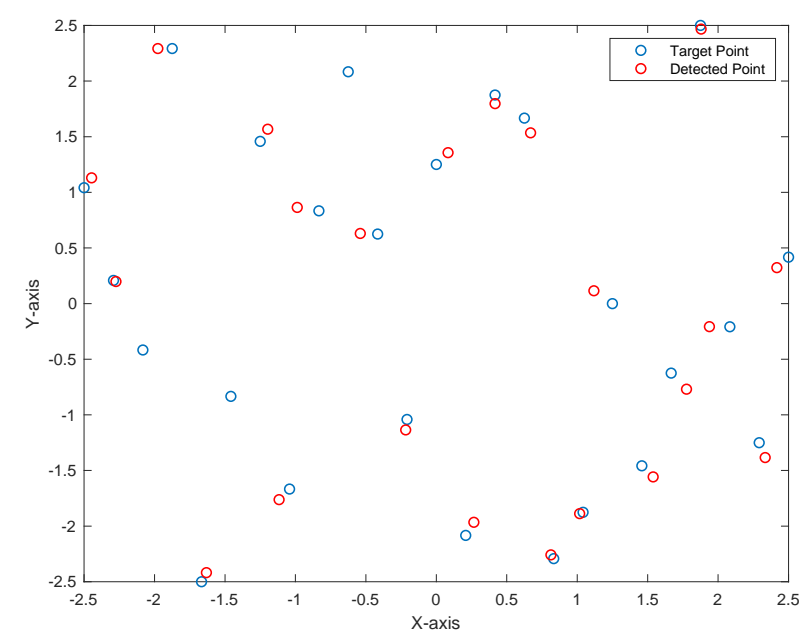


Figure 6. Result of random point detection using 6 LEDs.

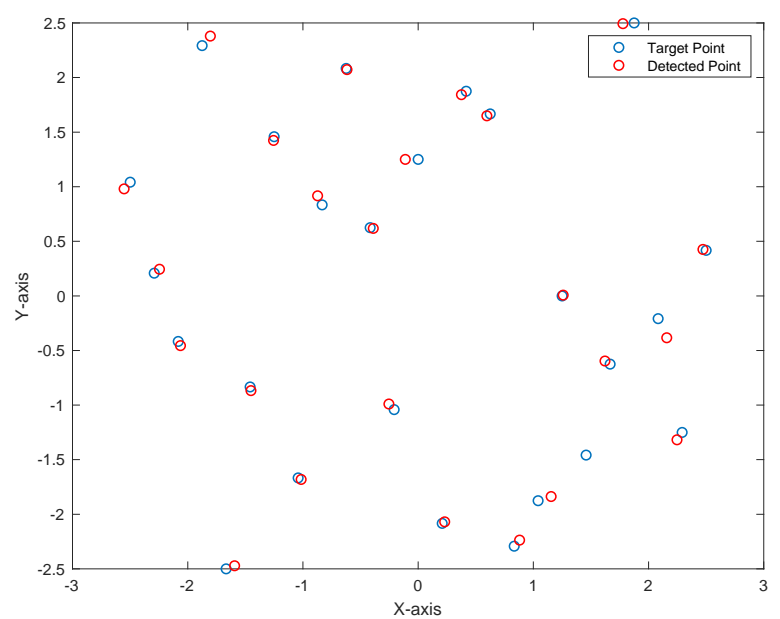

Figure 7. Result of random point detection using 8 LEDs.

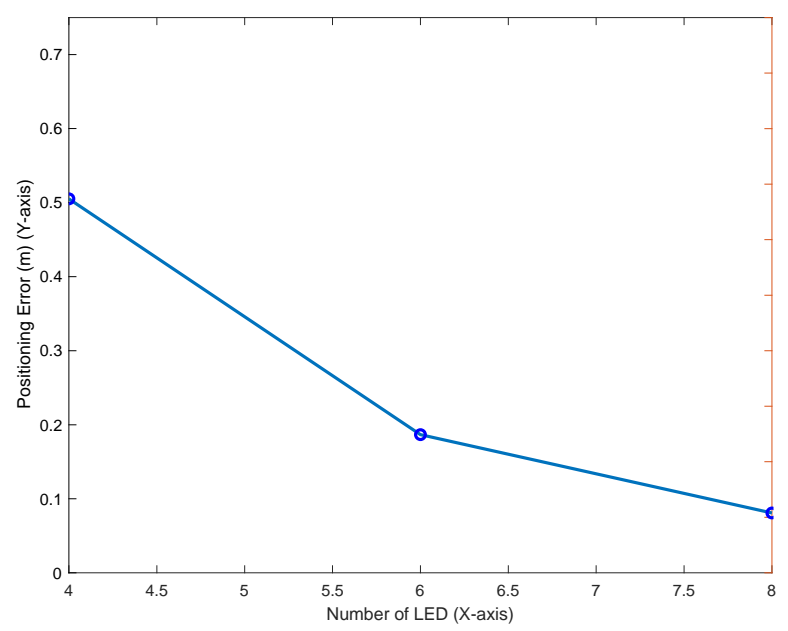

Figure 8. Comparison of positioning error.

From the results presented in Figure 8, it can be analyzed that in a system with 4 LEDs the positioning error is 0.505 meters, a system with 6 LEDs has a positioning error of 0.187 meters and a system with 8 LEDs has a positioning error of 0.081 meters. In general, the more LED lights that are installed on the room ceiling, the higher the accuracy value. An incremental linear curve can be obtained from the results of 4, 6 and 8 LEDs scenarios. We also found that the error at the detected position decreases as the LED light increased.

\section{Performance of Accuracy}

We evaluate the simulation accuracy of the system. This is measured by comparing the accuracy of the $\mathrm{x}$ and $\mathrm{y}$ coordinates detected by the original and $x$ and $y$ coordinates of the system. The accuracy of each simulation that is carried out depends on the number of the LEDs used. The higher number of LEDs used, the higher the accuracy so that the system has a better performance. Figure 9 shows that if more LEDs are used, the more accurate the positioning system will be. In a system with 4 LEDs the accuracy is $72.22 \%$, a system with 6 LEDs has an accuracy of $88.42 \%$ and a system with 8 LEDs has an accuracy of $96.62 \%$.

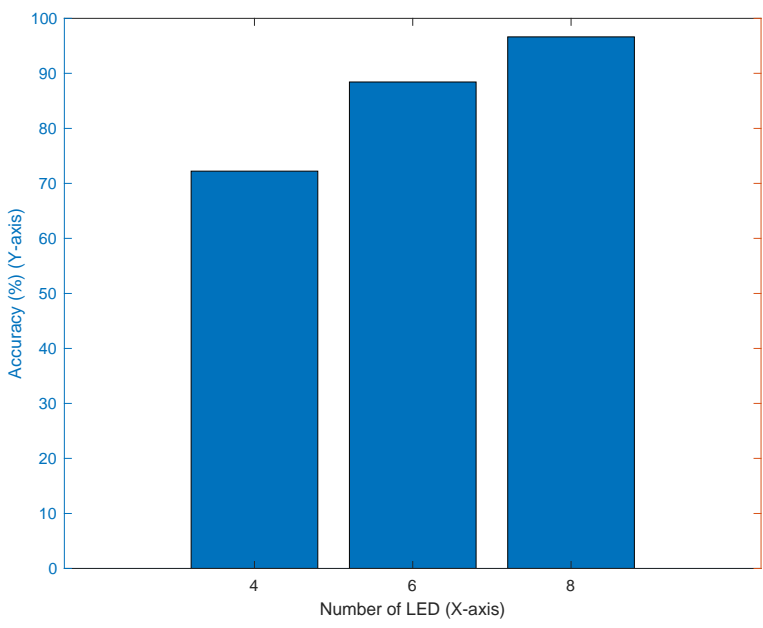

Figure 9. Comparison of accuracy score.

This research proves that the increases in the number of LEDs will increase the accuracy and reduce the positioning error. In the previous research, the researcher focused on increasing the accuracy with a different method.

\section{CONCLUSION}

This research conducted a performance study of the VLC-based positioning system using the RSS method in a closed room. The RSS method is used in positioning systems because of the low difficulty level and high accuracy. The simulation results show that the increasing number of LEDs used in the system result to an increasing number of random points detected, which increases the accuracy of the receiver's position and reduces the number of positioning errors.

\section{REFERENCES}

[1] S. Yang, E. Jung and S. Han, "Indoor Location Estimation Based on LED Visible light communication using multiple optical receivers," IEEE Communications Letters, vol. 17, no. 9, pp. 1834-1837, September 2013.

[2] H. Lv, L. Feng, A. Yang, P. Guo, H. Huang, and S. Chen, "High accuracy VLC indoor positioning system with differential detection," IEEE Photonics Journal, vol. 9, no. 3, pp. 1-13, 2017.

[3] K. Okusa and T. Kamakura, "Indoor location estimation based on the rss method using radial log-normal distribution," 16th IEEE International Symposium on Computational Intelligence and Informatics (CINTI), 2015, pp. 29-34.

[4] S. Shawky, M. A. El-Shimy, Z. A. El-Sahn, M. R. Rizk, and M. H. Aly, "Improved VLC-based indoor positioning system using a regression approach with conventional RSS techniques," in 2017 13th International Wireless Communications and Mobile Computing Conference (IWCMC), 2017, pp. 904-909.

[5] Z. Cui, Y. Wang and X. Fu, "Research on indoor positioning system based on VLC," 2020 Prognostics and Health Management Conference (PHM-Besançon), 2020, pp. 360-365.

[6] J. Wang, H. Li, X. Zhang and R. Wu, "VLC-based indoor positioning algorithm combined with OFDM and particle filter," in China Communications, vol. 16, no. 1, pp. 86-96, Jan. 2019.

[7] O.R.Popoola,S.Sinanovic',W.O.Popoola, and R.Ramirez-Iniguez, "Optical boundaries for led-based indoor positioning system," Computation, vol. 7, no. 1, p. 7, 2019. 
[8] D. Darlis, A. R. Darlis, and M. H. Abibi, "Implementasi sistem penyiaran musik digital di kafe menggunakan visible light communication," TELKOMIKA: Jurnal Teknik Energi Elektrik, Teknik Telekomunikasi, \& Teknik Elektronika, vol. 5, no. 1, p. 60, 2017.

[9] Z. Ghassemlooy, W. Popoola, and S. Rajbhandari, Optical Wireless Communications: System and Channel Modelling with MATLAB®. CRC press, 2019.

[10] C. Wang, L. Wang, X. Chi, S. Liu, W. Shi, and J. Deng, "The research of indoor positioning based on visible light communication," China Communications, vol. 12, no. 8, pp. 8592, 2015 . 